\title{
Model Limitations and Parameter-Estimation Methods for Agricultural Applications of Polarimetric SAR Interferometry
}

\author{
Juan M. Lopez-Sanchez, Senior Member, IEEE, J. David Ballester-Berman, and Yolanda Marquez-Moreno
}

\begin{abstract}
Application of polarimetric synthetic aperture radar interferometry to the retrieval of geophysical parameters from vegetated scenes is based on simple direct models of such scenes. The first part of this paper presents an analysis of the correspondence between these simple models, namely, the random volume over ground and the oriented volume over ground (OVoG), and experimental data from samples of two agricultural crops (maize and rice) acquired in controlled conditions. Although an overall agreement between model and data is clear, some discrepancies have been found as a consequence of two assumptions in the model formulation: vertical homogeneity of the vegetation volume and absence of multiple scattering effects inside the volume. This paper presents the shape and location of the visible region of the experimental coherences on the complex plane and compares it with the feasible region predicted by the model. This comparison has also pointed out the low sensitivity of the direct model to extinction coefficients. In the second part, two different strategies for a complete inversion (i.e., estimation of all model parameters) of the OVoG model are proposed and compared, using the same data set. The first one is based on a combination of geometrical and numerical approaches (genetic algorithms) and the second one on a dual-baseline configuration. In all cases, ground topography is accurately estimated, with a maximum error of $10 \mathrm{~cm}$. Vegetation height estimates are accurate up to $30 \mathrm{~cm}$, with some bands and baseline configurations providing errors below $15 \mathrm{~cm}$. However, results obtained for the extinction coefficients are not stable with frequency and exhibit high variability.
\end{abstract}

Index Terms-Agriculture, dual baseline, genetic algorithms, parameter retrieval, polarimetric synthetic aperture radar interferometry, vegetation.

\section{INTRODUCTION}

$\mathbf{T}$ HE ESTIMATION of biophysical parameters of vegetation cover from radar remote sensors with interferometric and polarimetric capabilities [polarimetric interferometric synthetic aperture radar (PolInSAR)] has been demonstrated recently at different frequencies: P-band [1], L-band [2], [3], and even X-band [4]. In the case of forest cover, the random volume over ground (RVoG) model proposed in [5] and [6] has been successfully inverted for forest height and biomass [3], [7]. Instead, when working with agricultural crops, the vertical orientation of the stems and other preferred orienta-

Manuscript received September 16, 2006; revised March 22, 2007. This work was supported by the Spanish Ministry of Education and Science (MEC) and EU FEDER under Project TEC2005-06863-C02-02, and by the Generalitat Valenciana under Project ACOMP07-087.

The authors are with the Signals, Systems, and Telecommunication Group, University of Alicante, 03080 Alicante, Spain (e-mail: juanma-lopez@ieee.org; davidb@dfists.ua.es; yolanda@dfists.ua.es).

Digital Object Identifier 10.1109/TGRS.2007.900690 tions of different plant parts suggest that the best candidate model corresponds to an oriented VoG (OVoG) [5], [6], [8]. To date, only partial inversion of the OVoG model has been achieved, since it has been employed to retrieve only the ground topography and the vegetation height of agricultural crops, obtaining precise estimates in laboratory conditions with samples of maize and rice [9]. Despite the apparent necessity of modeling the orientation of the plants, satisfactory results were also obtained by applying the RVoG model in these crop experiments. Note that, in both RVoG and OVoG cases, all data analyses and retrieval experiments have been carried out by assuming vertically homogeneous volumes.

The first objective of this paper is to establish a comparison between the radar observables and the predictions of the theoretical direct models (RVoG and OVoG). To do that, the knowledge of the ground truth of the two crop samples will be used to compute the positions of the interferometric coherences on the complex plane, which are also known as coherence loci. These coherence loci will be compared against those obtained from the experimental data. Conclusions about model limitations will be derived from this analysis.

In the second part of this paper, we focus on several approaches for estimating the full set of parameters of the OVoG model. As already noted in the literature [9], [10], the use of a single-baseline PolInSAR configuration leads to an indeterminate system of equations: There are seven unknowns and only six observables. Here, we propose first a two-step hybrid procedure to solve this problem. The first step is based on the assumption of a linear distribution of the coherences on the complex plane (which is tested in the first part of this paper). Under this assumption, the line fitting [7] commonly used for inverting the RVoG model provides an estimation of the ground topography (one of the unknowns). Then, the second stage consists of a numerical optimization algorithm for inverting the remaining problem with six observables and six unknowns. Finally, a second inversion scheme, based on a dual-baseline configuration is also tested with the same purpose. Limitations and potentials of both techniques are discussed.

The sensitivity of the model to the parameters is a key issue that will be present in all the analyses. It will be shown that extinction coefficients do not produce clear signatures in the model deliverables, thus limiting its applicability to parameter inversion. In addition, the model is also very simple from the electromagnetic point of view since the interaction between the vegetation elements is simplified in the formulation. 


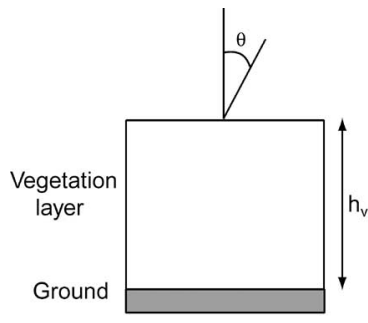

(a)

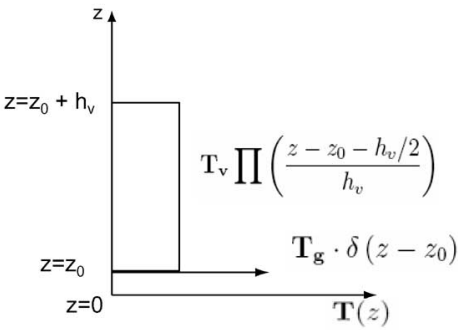

(b)
Fig. 1. Two-layer vegetation model. (a) Geometry of a vegetation volume over the ground. (b) Vertical profile of the coherence function defined in (4) according to the framework proposed in [3]. $z$ denotes the vertical coordinate, $h_{\mathrm{v}}$ is the vegetation height (i.e., the depth of the layer), and $\theta_{0}$ is the mean incidence angle.

This paper is organized as follows. Section II reviews the formulation of the model and highlights some important aspects of the complex coherence loci predicted by the OVoG. A comparison between real data and model predictions is shown in Section III. A study of the inversion strategies is described in Section IV. Finally, conclusions are summarized in Section V.

\section{MOdEl Formulation}

\section{A. Direct Model}

Although it is available in the literature, for readiness purposes, this section presents a brief summary of the formulation of the PolInSAR observables and the simple models used to describe a scene with vegetation.

The model of the vegetation scene, both in RVoG and OVoG versions, considers the target as a two-layer scene, as shown in Fig. 1(a). This vegetation model has been proposed and used in [5], [6], and [8] to rigorously derive an analytical expression of the complex interferometric coherence, and any interested reader can consult these references for detailed explanations. For the sake of conciseness, in this paper, we adopt a simplified interpretation to arrive to the same final formulation, which was proposed in [7]. Assuming that the scattering is equivalent at both ends of the baseline, the complex interferometric coherence $\gamma$ can be generalized to incorporate the polarimetric information, yielding the following expression [11]:

$$
\gamma=\frac{\mathbf{w}^{* T} \boldsymbol{\Omega}_{12} \mathbf{w}}{\mathbf{w}^{* T} \mathbf{T}_{11} \mathbf{w}}
$$

where w's are unitary vectors employed to select a certain polarization combination, and $\mathbf{T}_{11}$ and $\boldsymbol{\Omega}_{12}$ matrices are expressed for the RVoG and OVoG models as follows:

$$
\begin{aligned}
\mathbf{T}_{11}= & e^{-\frac{\sigma_{T}(\mathbf{w}) h_{\mathrm{v}}}{\cos \theta_{0}}} e^{-\frac{\sigma_{R}(\mathbf{w}) h_{\mathrm{v}}}{\cos \theta_{0}}} \int_{z} e^{\frac{\sigma_{T}(\mathbf{w})+\sigma_{R}(\mathbf{w})}{\cos \theta_{0}} z} \mathbf{T}(z) d z \\
\boldsymbol{\Omega}_{12}= & e^{j \phi_{0}} e^{-\frac{\sigma_{T}(\mathbf{w}) h_{\mathrm{V}}}{\cos \theta_{0}}} e^{-\frac{\sigma_{R}(\mathbf{w}) h_{\mathrm{V}}}{\cos \theta_{0}}} \\
& \times \int_{z} e^{\left(\frac{\sigma_{T}(\mathbf{w})+\sigma_{R}(\mathbf{w})}{\cos \theta_{0}}+j k_{z}\right) z} \mathbf{T}(z) d z
\end{aligned}
$$

where all parameters are defined in [7] and [8].
In this simplified framework, the total contribution of the scene to the matrices $\mathbf{T}_{11}$ and $\boldsymbol{\Omega}_{12}$ in (2) and (3) is obtained by means of an integral along the vertical coordinate $z$ of the scattering properties of the scene. To this end, a vertical profile of the coherence matrix properties $\mathbf{T}(z)$ is defined. The shape of $\mathbf{T}(z)$ is shown in Fig. 1(b) and can be expressed as

$$
\mathbf{T}(z)=\mathbf{T}_{\mathrm{g}} \delta\left(z-z_{0}\right)+\mathbf{T}_{\mathrm{v}} \Pi\left(\frac{z-z_{0}-h_{\mathrm{v}} / 2}{h_{\mathrm{v}}}\right)
$$

where $\mathbf{T}_{\mathrm{g}}$ and $\mathbf{T}_{\mathrm{v}}$ are the contributions from the ground and the volume, respectively [7]. Note that, in this formalism of the 1-D profile, $\mathbf{T}_{\mathrm{v}}$ is dimensionless, whereas $\mathbf{T}_{\mathrm{g}}$ presents dimensions of length since it will result in the integral of the delta function.

The ground is assumed to contribute with a localized backscattering response with a scattering phase center at the ground interface, whereas the volume response is uniformly distributed over the whole vegetation depth. Note that the ground contribution may correspond to a direct return from the ground or a ground-trunk interaction. These assumptions, which are important for a simple formulation, will be the origin of some of its limitations. In fact, recent investigations [12] have demonstrated that these hypotheses are only partially valid.

As known [8], in the case of an oriented volume, the only polarizations that do not change as the wave travels into the volume are the eigenpolarizations, which are generally two orthogonal polarizations that correspond to the maximum and minimum values of the extinction coefficient. Accordingly, the expressions in (2) and (3) are also formally correct for the eigenpolarizations if an oriented volume is present. Since most agricultural plants exhibit a preferred vertical orientation, mainly dominated by the stems, the eigenpolarizations can be identified as vertical and horizontal polarizations. In such a situation, the three polarimetric channels corresponding to the formulation in (2) and (3) are $\mathrm{HH}, \mathrm{HV}$, and VV.

After some manipulations described in [7] and [9], the interferometric coherence can be expressed as

$$
\begin{aligned}
\gamma & =e^{j \phi_{0}} \cdot \frac{\mu\left(\sigma_{\mathbf{w}}, \mathbf{w}\right)+\gamma_{\mathbf{v}}\left(\sigma_{\mathbf{w}}\right)}{1+\mu\left(\sigma_{\mathbf{w}}, \mathbf{w}\right)} \\
& =e^{j \phi_{0}} \cdot\left[\gamma_{\mathbf{v}}\left(\sigma_{\mathbf{w}}\right)+\frac{\mu\left(\sigma_{\mathbf{w}}, \mathbf{w}\right)}{1+\mu\left(\sigma_{\mathbf{w}}, \mathbf{w}\right)}\left(1-\gamma_{\mathbf{v}}\left(\sigma_{\mathbf{w}}\right)\right)\right] \\
& =e^{j \phi_{0}} \cdot\left[\gamma_{\mathbf{v}}\left(\sigma_{\mathbf{w}}\right)+L\left(\sigma_{\mathbf{w}}, \mathbf{w}\right)\left(1-\gamma_{\mathbf{v}}\left(\sigma_{\mathbf{w}}\right)\right)\right]
\end{aligned}
$$

where the equivalence $2 \sigma_{\mathbf{w}}=\sigma_{T}(\mathbf{w})+\sigma_{R}(\mathbf{w})$ has been introduced, $0 \leqslant L\left(\sigma_{\mathbf{w}}, \mathbf{w}\right) \leqslant 1, \gamma_{\mathrm{v}}\left(\sigma_{\mathbf{w}}\right)$ denotes the volume coherence, and $\mu\left(\sigma_{\mathbf{w}}, \mathbf{w}\right)$ represents the ground-to-volume ratio. The following two definitions have been used (see [7] and [9] for details about these expressions)

$$
\begin{aligned}
\gamma_{\mathbf{v}}\left(\sigma_{\mathbf{w}}\right) & =\frac{2 \sigma_{\mathbf{w}}}{\cos \theta_{0}\left(e^{\frac{2 \sigma_{\mathbf{w}} h_{\mathbf{v}}}{\cos \theta_{0}}}-1\right)} \cdot \frac{e^{\left(\frac{2 \sigma_{\mathbf{w}}}{\cos \theta_{0}}+j k_{z}\right) h_{\mathrm{v}}}-1}{\frac{2 \sigma_{\mathbf{w}}}{\cos \theta_{0}}+j k_{z}} \\
\mu\left(\sigma_{\mathbf{w}}, \mathbf{w}\right) & =\frac{\mathbf{w}^{* T} e^{\frac{-2 \sigma_{\mathbf{w}} h_{\mathrm{v}}}{\cos \theta_{0}}} \mathbf{T}_{\mathrm{g}} \mathbf{w}}{\mathbf{w}^{* T} \frac{\cos \theta_{0}}{2 \sigma_{\mathbf{w}}}\left(1-e^{\frac{-2 \sigma_{\mathbf{w}} h_{\mathbf{v}}}{\cos \theta_{0}}}\right) \mathbf{T}_{\mathbf{v}} \mathbf{w}}
\end{aligned}
$$




\section{B. Basic Inversion Approaches}

The observables delivered by a polarimetric interferometer are the complex coherences for the three channels in linear basis $(\mathrm{HH}, \mathrm{HV}$, and $\mathrm{VV})$, summing a total of six real data (real and imaginary parts, or amplitudes and phases). In this paper, we are not interested in pure polarimetric ratios (i.e., zero-baseline configurations) nor in polarization choices where the polarizations are different at the ends of the baseline. An analysis about the potential contribution of such observables to this application can be consulted in [6].

If the scene corresponds to the RVoG model, where the extinction does not depend on polarization $\left(\sigma_{\mathbf{w}}=\sigma\right.$ for all polarizations), the model is fully described by six parameters: topographic phase $\phi_{0}$, vegetation depth $h_{\mathrm{v}}$, extinction $\sigma$, and ground-to-volume ratios for the three channels, i.e., $\mu_{\mathrm{HH}}, \mu_{\mathrm{HV}}$, and $\mu_{\mathrm{VV}}$. In that case, a numerical method for inverting the model can be implemented (e.g., by minimizing the distance between observations and model predictions), since the number of independent observations is equal to the number of parameters (unknowns). The conditioning and uniqueness of this inversion problem were addressed in [13, Sec. 4.A], and they rely on the separation of the effective scattering centers of the selected polarizations. Although it is not formally necessary, the numerical inversion is easier if one chooses polarizations with clear separations, which can be decided on a physical basis (e.g., Pauli basis provides direct scattering $\mathrm{HH}+\mathrm{VV}$ located at the volume and dihedral scattering $\mathrm{HH}-\mathrm{VV}$ located at the ground position) or on mathematical foundations (e.g., polarizations that optimize the coherence [11]). This freedom in the polarization selection is based on the independence of extinction with respect to polarization, because the formulation and the inversion remain unchanged when one substitutes $\mathrm{H}$ and $\mathrm{V}$ by polarizations 1 and 2, whatever they are. On the other hand, this nonlinear problem has, in general, multiple solutions due to its intrinsic nature: Different combinations of vegetation height and extinction provide the same observables. Therefore, any numerical solution must consider these aspects to ensure that the obtained solution is the true one or the closest.

Note that a complementary approach would consist in increasing the number of polarimetric channels employed in the inversion (not only three), since each additional channel provides two real data (real and imaginary parts of the coherence) and only one extra unknown (the corresponding ground-tovolume ratio). In this case, although the added channels are not obtained from independent measurements, a more accurate estimation of the topographic phase can be performed since the line fitting would be applied more robustly and, additionally, the numerical optimization procedure performed for calculating the six remaining unknowns would become less sensitive to noise.

When the scene corresponds to the OVoG model (extinction depends on wave polarization), there appears an indetermination in the problem. The observation space is the same as before (six real data), but there are two different extinctions to be determined $\left(\sigma_{\mathrm{H}}\right.$ and $\left.\sigma_{\mathrm{V}}\right)$, thus increasing the number of unknowns to seven. In addition, the formulation of the model is suited only for the eigenpolarizations ( $\mathrm{H}$ and $\mathrm{V}$ ), so, in principle, the rest of the polarizations cannot be used to enlarge the observation space as before. As a consequence of the mandatory use of the eigenpolarizations, in the OVoG, we cannot choose the best polarization combination on the basis of their separation on the complex plane. Therefore, the problem of this inversion approach is twofold: there are more unknowns than measured data, and the scattering centers corresponding to these data (whose positions depend on the scene and the interferometer configuration) may not be particularly well separated in height, since we cannot select other polarizations different from the eigenpolarizations.

The retrieval of the full set of parameters that appear in the OVoG model will be treated in Section IV, where several inversion approaches will be proposed and compared. Before that, the next section is focused on the fidelity and sensitivity of the direct model when describing the PolInSAR response of an agricultural sample.

\section{COMParison OF Direct Models WITH EXPERIMENTAL DATA}

The analysis is based on the representation on the complex plane of the coherences defined in (5). Note that this representation leads to a clear and powerful physical interpretation of the model, which, in turn, has significantly helped the subsequent inversion approach [7], [13].

The possible positions of the complex coherences on the complex plane are shown in Fig. 2 for a typical configuration in two cases: an RVoG with an extinction of $1 \mathrm{~dB} / \mathrm{m}$, and an OVoG with extreme extinctions of 0 and $3 \mathrm{~dB} / \mathrm{m}$, respectively. Note that a repeat-pass or alternate-transmit system is assumed. In the random volume case, extinction does not depend on polarization, and all coherences [shown by crosses in Fig. 2(a)] corresponding to $\mathrm{w}$ polarization selections lie along a single straight line on the unit circle. The exact position along the line is defined by the ground-to-volume ratio $\mu$, which changes as a function of the polarization channel. The line departs from the $\exp \left(j \phi_{0}\right)$ point in the unit circumference when $\mu \rightarrow \infty$ (i.e., the response from the ground completely dominates the backscattering from the target).

Instead, when an oriented volume is present, we only have defined three possible interferometric coherences (two copolar and one crosspolar), obtained for each possible combination of eigenpolarizations in transmission and reception, which are denoted as channels 1 and 2 in Fig. 2(b). The three coherences, which are represented by stars in Fig. 2(b), do not lie on a line on the complex plane. Moreover, for a given scene, one cannot change the ground-to-volume ratios of the selected channels, and consequently, the concept of line to describe the positions of coherences of an oriented volume does not apply in this case. Notwithstanding, with respect to the relative position of the three coherences, one can draw three lines starting from the topographic phase point $\exp \left(j \phi_{0}\right)$ and crossing the coherences. The most external line corresponds to the eigenpolarization channel with the lowest extinction, whereas the inner line corresponds to the highest extinction channel. However, due to the aforementioned inability to generate lines by changing the ground-to-volume ratios, none of these lines should be confused with the one appearing in Fig. 2(a). 


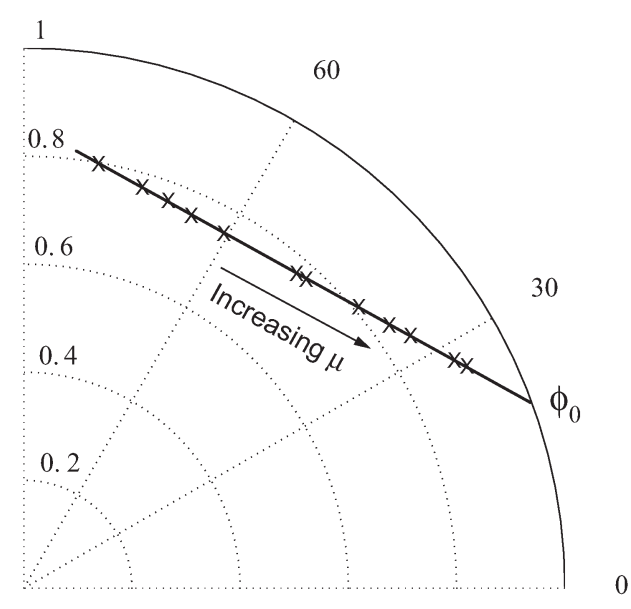

(a)

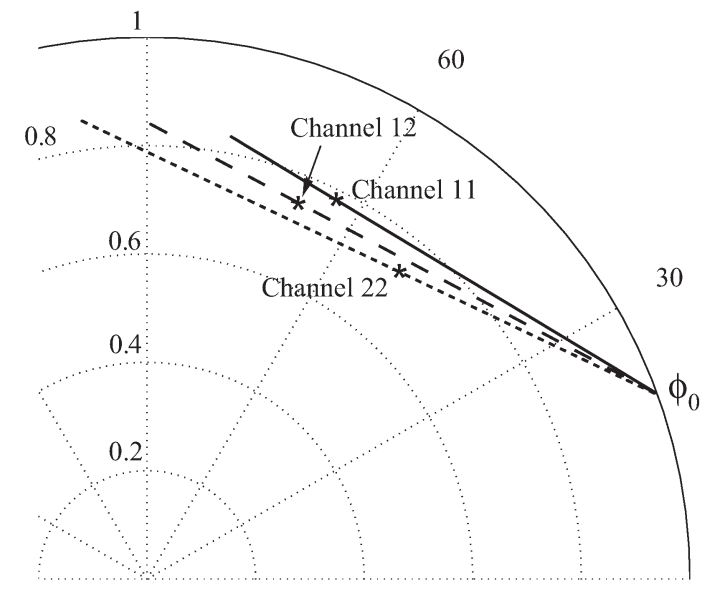

(b)

Fig. 2. Positions of the coherences on the complex plane. Parameters: $h_{\mathrm{v}}=$ $2 \mathrm{~m}, \phi_{0}=20^{\circ}$, and $k_{z}=1.04$. (a) RVoG with $\sigma=1 \mathrm{~dB} / \mathrm{m}$. Crosses "x" denote different coherences obtained from different polarization channels. (b) OVoG with $\sigma_{1}=0 \mathrm{~dB} / \mathrm{m}$ and $\sigma_{2}=3 \mathrm{~dB} / \mathrm{m}$. Stars "**" denote the only three possible coherences for the eigenpolarization combinations (11, 12, and 22). Lines are drawn from the topographic phase point for interpretation purposes.

The separation between the lines drawn for the OVoG case [Fig. 2(b)] depends both on the target and on the interferometric setup. First, the separation is directly proportional to the differential extinction coefficient $\sigma_{\text {diff }}$, which is defined as the difference between the two extinction coefficients at the eigenpolarizations: $\sigma_{\text {diff }}=\sigma_{2}-\sigma_{1}$. Second, the separation is also controlled by the product $k_{z} \cdot h_{\mathrm{v}}$, which, in turn, depends on the frequency, the baseline, and the depth of the vegetation layer. If the differential extinction and the $k_{z} \cdot h_{\mathrm{v}}$ product are maintained below a certain threshold, the region occupied by the three lines is so narrow that it can be treated as a single line, as in the case of the RVoG model. This feature has been used in [9] to derive a partial inversion scheme for the OVoG, based on the original one for the RVoG model [7].

Next, we compare the feasible loci with experimental coherences obtained from the two crop samples of maize and rice, measured at the European Microwave Signature Laboratory (EMSL), Joint Research Centre-Ispra. The height of the maize plants is $1.80 \mathrm{~m}$, whereas the rice plants are $75 \mathrm{~cm}$ high.
The required multilooking is performed by averaging the data acquired at different azimuth angles (by rotating the platform) and with adjacent frequency bands. For example, for the maize target, we use 72 azimuth angles and nine bands; therefore, a total of 648 independent samples are averaged. More details about the experiment configuration and the ground truth can be consulted in [9]. Fig. 3(a) shows the position of the coherences on the complex plane for a particular combination of frequency ( $3 \mathrm{GHz})$ and baseline $\left(0.5^{\circ}, k_{z}=1.56\right)$. In this case, the product $k_{z} \cdot h_{\mathrm{v}}$ is 2.81 . We present the coherences in linear basis (VV, $\mathrm{HH}$, and $\mathrm{HV}$, which correspond to the eigenpolarizations for a vertically oriented volume), Pauli basis, and the optimized ones [11]. Then, the true topographic phase $\phi_{0}$, which is known in the experiment, is used in Fig. 3(b) to obtain the extreme lines that should enclose all the linear coherences ( $\mathrm{HH}, \mathrm{VV}$, and $\mathrm{HV})$, according to the OVoG direct model. The lowest extinction has been chosen as $0 \mathrm{~dB} / \mathrm{m}$ for simplicity.

Due to the vertical orientation of the plants, it is clear that vertical polarization exhibits the greatest extinction, therefore, the line corresponding to $\mathrm{VV}$ is the closest to the origin. Accordingly, the $\mathrm{HH}$ coherence line would be the farthest one from the origin because horizontal polarization suffers from the lowest extinction. Note that the relative position of these extreme lines does not imply that the actual $\mathrm{HH}$ coherence is higher than the actual VV coherence, because these coherences are just two points (one for each channel), and the corresponding ground-tovolume at each channel is the parameter that drives the actual position along the mentioned lines. In other words, there are many points in the inner line with higher coherence than many points in the outer one.

However, in opposition to the argument of the extreme lines for the copolar channels, one can observe in Fig. 3(b) that the line crossing the $\mathrm{HV}$ coherence, not the $\mathrm{HH}$ one, is the farthest from the origin. We have also observed this behavior at other frequencies and in the data acquired for the rice sample.

In second place, since we know that the vegetation height is $1.80 \mathrm{~m}$, we can also overlay the region of lines corresponding to this height. To do that, we assume extinctions ranging from 0 to $5 \mathrm{~dB} / \mathrm{m}$, which are the typical values for this crop [14] assuming a similar development stage and analogous environmental conditions. When we do that [see Fig. 3(c)], the region defined by the model does not match the experimental data at all. Only if we reduce the vegetation depth down to $1.50 \mathrm{~m}$ and extend the maximum extinction to $9 \mathrm{~dB} / \mathrm{m}$ [see Fig. 3(d)], the modeled and the experimental regions overlap. Recent investigations with the same data set [12] have shown that the volume scattering shows a maximum response from the center of the vegetation layer and not from the top, whereas these models assume that the maximum scattering always arises from the top layer, with an exponential reduction of scattering due to extinction inside the medium. Consequently, the lower position of the backscattering peak may partially explain why we have to reduce the effective height to get the fit between observations and model predictions.

Additionally, it is also very clear that a change in several decibels per meter in extinction only produces a small displacement in the coherence position. The low sensitivity of the model to this important parameter can be understood from the 


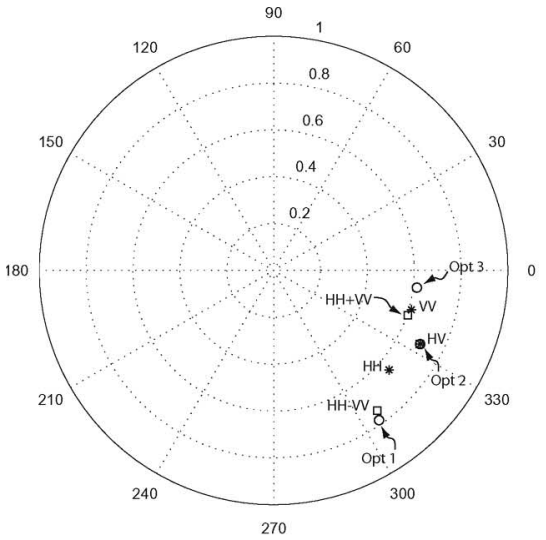

(a)

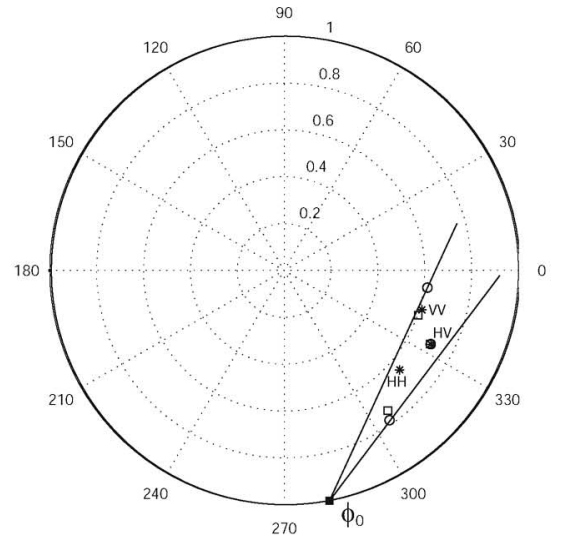

(b)

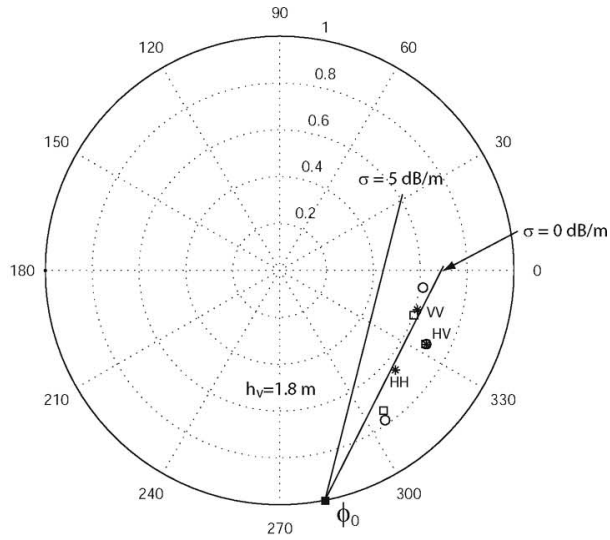

(c)

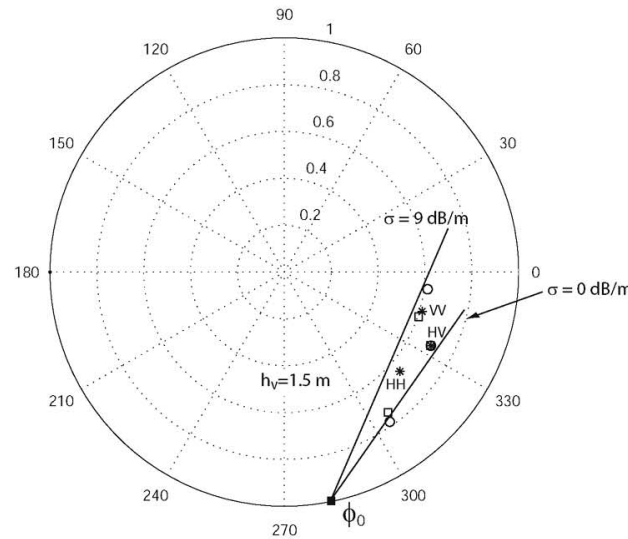

(d)

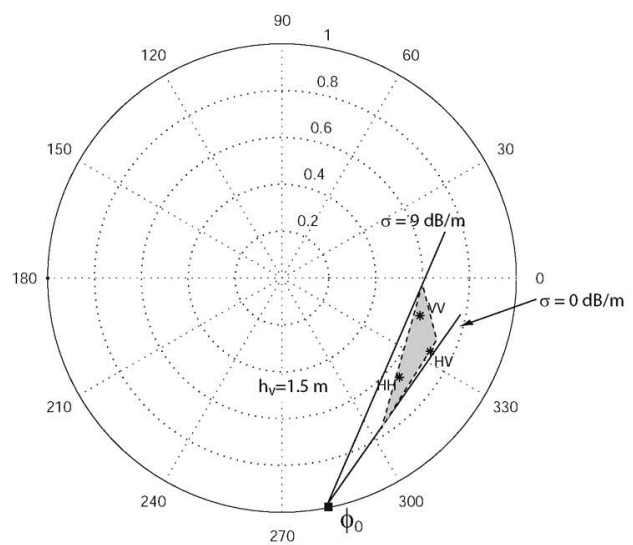

(e)

Fig. 3. Experimental coherences on the complex plane for the maize sample. Parameters: $f=3 \mathrm{GHz}, B=0.5^{\circ}$, and $k_{z}=1.56$. (a) Coherences for linear basis, Pauli basis, and optimized ones. (b) Possible lines from the exact topographic phase. (c) Overlay of the theoretical region with $h_{\mathrm{v}}=1.80$. (d) Overlay of the theoretical region with $h_{\mathrm{v}}=1.50$. (e) Overlay of the visible and theoretical regions with $h_{\mathrm{v}}=1.50$.

mathematical expression of the coherence in (5)-(7). Extinction is almost always multiplied by $h_{\mathrm{v}}$ in this formulation, therefore, the derivative of coherence with respect to extinction is approximately proportional to $h_{\mathrm{v}}$. Since we are dealing with short vegetation scenes (up to $2 \mathrm{~m}$ ), the derivative is small and, as a result, the sensitivity to extinction is poor. This will complicate the robustness of the model inversion, as we will show in the next section.

It is also interesting to observe the region occupied by other possible coherences derived from the data. We have computed all possible $\mathrm{w}$ vectors, and they have been substituted into (1) to find other possible coherences from the data. The length and width of the resulting region, which is shown in Fig. 3(e), provide an idea of the conditioning of the inversion problem. The narrower and longer the region, the easier would be the solution (at least for the topographic phase in the hybrid approach proposed in the next section). This triangular region can be named as visible region, which is analogous to the visible line length defined in [7]. Note that the geometrical solution of the problem for $\phi_{0}$ and $h_{\mathrm{v}}$, by assuming a known range of extinctions, is presented in [9].

An inspection of the data at other frequencies and baselines reveals that the matching between the model and the experimental data is never perfect. Fig. 4(a) shows the visible region for the maize at $6 \mathrm{GHz}$ with a baseline of $0.25^{\circ}$ (same $k_{z}=1.56$ as in Fig. 3). In this case, we can match the visible region with the theoretical model by choosing $h_{\mathrm{v}}=1.7 \mathrm{~m}$ and an extreme extinction of $3 \mathrm{~dB} / \mathrm{m}$. Repeating the same reasoning for the rice sample, we obtain the representation of Fig. 4(b) at $8 \mathrm{GHz}$ with a $0.5^{\circ}$ baseline $\left(k_{z}=4.16\right)$. We have to choose an extinction of $12 \mathrm{~dB} / \mathrm{m}$ and a height of $0.62 \mathrm{~m}$ to accommodate the rice data inside the model predictions. Nevertheless, in this case, all coherences remain concentrated on a small cluster close to the unit circumference, so the sensitivity of the model to its parameters is quite limited.

The discrepancies between the model predictions and the experimental data can be explained by several characteristics of the model, which make it particularly simple. First of all, the vegetation layer is assumed to be homogeneous, and it is not true. For instance, the maize plants do not bear leaves in their lower part (about $40 \mathrm{~cm}$ from the ground). In the case of rice, the stems depart from a point of the ground surface but they separate each other as one moves toward the upper part of the plants. The top part of the rice plants is bent. Consequently, it is clear that the true vegetation volume of maize and rice crops is not homogeneous, as it is assumed by the model.

In addition to the lack of geometrical homogeneity in the vertical distribution of the plant components, the model is also very simple from the electromagnetic point of view, since the interaction between the vegetation elements has been taken 


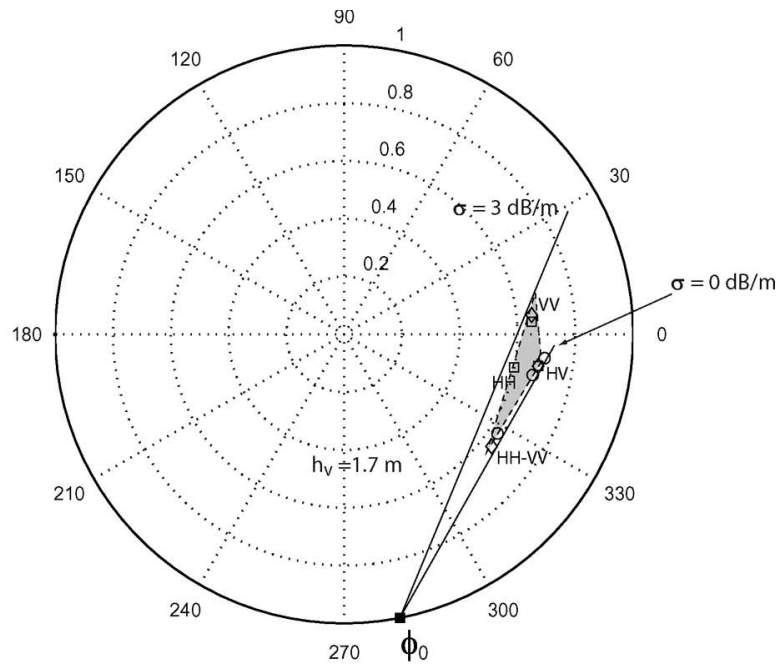

(a)

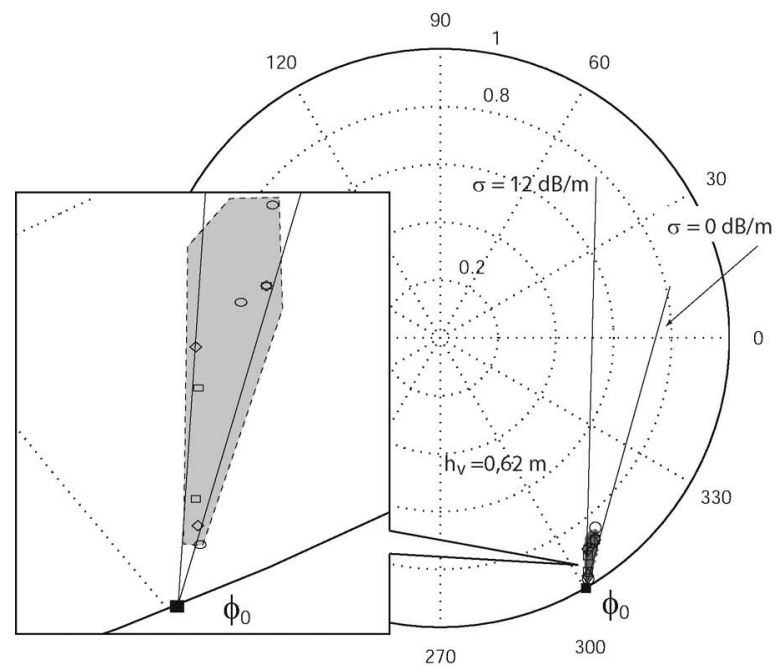

(b)
Model

parameters

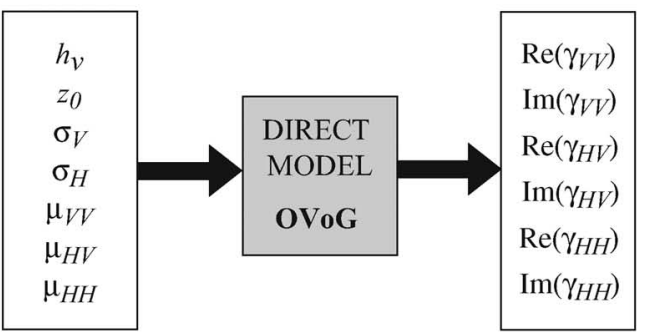

(a)

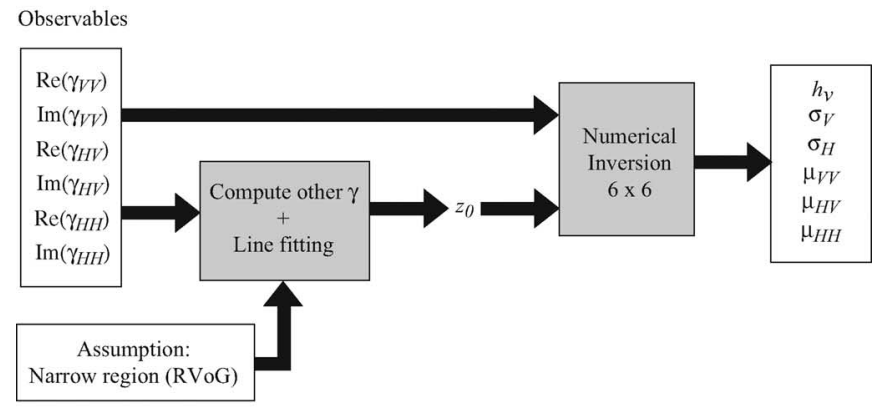

(b)

Observables

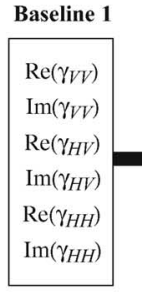

\begin{tabular}{c|c|c|c} 
Baseline 2 & Numerical & $\sigma_{V}$ \\
Inversion & \\
$12 \times 7$ & $\sigma_{H}$ \\
$\operatorname{Re}\left(\gamma_{V V}\right)$ & & $\mu_{V V}$ \\
$\operatorname{Im}\left(\gamma_{V V}\right)$ & & $\mu_{H V}$ \\
$\mu_{H H}$ \\
\end{tabular}

Fig. 4. Visible region of the coherences. (a) Maize sample ( $f=6 \mathrm{GHz}, B=$ $0.25^{\circ}$, and $\left.k_{z}=1.56\right)$ and (b) rice sample $\left(f=8 \mathrm{GHz}, B=0.5^{\circ}\right.$, and $k_{z}=$ 4.16).

into account only partially by means of a statistical modeling of the total first-order backscattering response of the medium. This interaction leads to multiple scattering effects, which have been demonstrated with simulations and experiments in literature. The presence of multiple scattering leads to important features in the backscattering response from such targets. At first instance, multiple scattering changes the total value of the radar cross section (RCS) of the plants. This was shown in [15], where coherent effects were noticed by simulating the electromagnetic response of rice fields with clusters of cylinders. Since the definition of the interferometric coherence normalizes the RCS, the influence of multiple scattering in the coherence (both in amplitude and phase) is not produced by the total RCS but by the vertical profile or distribution of RCS values. For example, the high-resolution radar images obtained in [16] for wheat samples illustrate the presence of secondorder volume scattering events. In particular, the commented anomaly in the HV coherence could be explained by the strong multiple scattering present at this channel.

\section{INVERSION STRATEGIES}

The situation of the OVoG direct model described so far corresponds to the block diagram shown in Fig. 5(a), where it is shown that the model is described by seven parameters, whereas only six data are measured in a single-baseline PolInSAR observation. Two inversion approaches for the estimation of the full set of parameters of the OVoG model are proposed in 
this section. The first one is a hybrid scheme consisting of the combination of the well-known line fitting of the coherences on the complex plane and a numerical optimization. The second inversion algorithm is based on extending the observation space with a dual-baseline configuration.

It is important to note that, in contrast to other inversion procedures [7], [9] where only the vegetation height and the ground topography are retrieved, both approaches proposed here provide, for the first time, the capability for the estimation of the whole set of parameters that describe the vegetation cover according to this model. Consequently, the results of this paper, if were linked to allometric and empirical equations, would provide improved and more complete information that describes crop development and status or even computes yield predictions.

\section{A. Hybrid (Geometrical-Numerical) Approach}

As already mentioned in Section III, when describing the OVoG direct model, the width of the visible region of the coherences on the complex plane is controlled by the differential extinction coefficient and by the product $k_{z} \cdot h_{\mathrm{v}}$. If both are maintained below a certain threshold, the region is so narrow that it can be treated as a single line, as in the case of the RVoG model. This feature was first used in [9] to derive a partial inversion scheme: The ground topography and the vegetation height were estimated, but assuming a priori known range of extinctions. Here, we extend this inversion as explained in the following. A block diagram is shown in Fig. 5(b).

The first stage of the algorithm consists of the same line fitting of the coherences, resembling their theoretical distribution for the RVoG case, already shown in Fig. 2. In order to help the fitting procedure, following the strategy in [7], we first compute other polarization states that are different from the original data in linear $\mathrm{H}-\mathrm{V}$ basis. Consequently, the line fitting is better determined with this enlarged set of points, although the system only provides three polarimetric channels $(\mathrm{HH}, \mathrm{VV}$, and $\mathrm{HV})$. We cannot state that this approach is strictly valid in the OVoG model, because the analytical expressions of the OVoG model are valid only for the eigenpolarizations. Anyway, if we overlook this theoretical limitation and calculate the coherences for the Pauli basis and the optimized channels, the experiments have shown that they still lie on a narrow region. Therefore, this extension has been used to ensure a good line fitting, thus provide an estimate of the topographic phase $\phi_{0}$. In summary, by assuming that the distribution of coherences is similar to that of the RVoG model, we estimate the topographic phase or, equivalently, the ground position $z_{0}$.

Once we know one of the seven parameters $\left(z_{0}\right)$, a numerical optimization algorithm is applied to find a solution for the remaining six parameters by using the set of six real values provided by the linear polarizations (i.e., real and imaginary parts of the coherences). Note that a different geometrical approach was introduced in [10], which relates these parameters with the angle formed by the lines corresponding to the eigenpolarizations.

The first results obtained with the proposed hybrid (geometrical-numerical) approach are described in the follow-
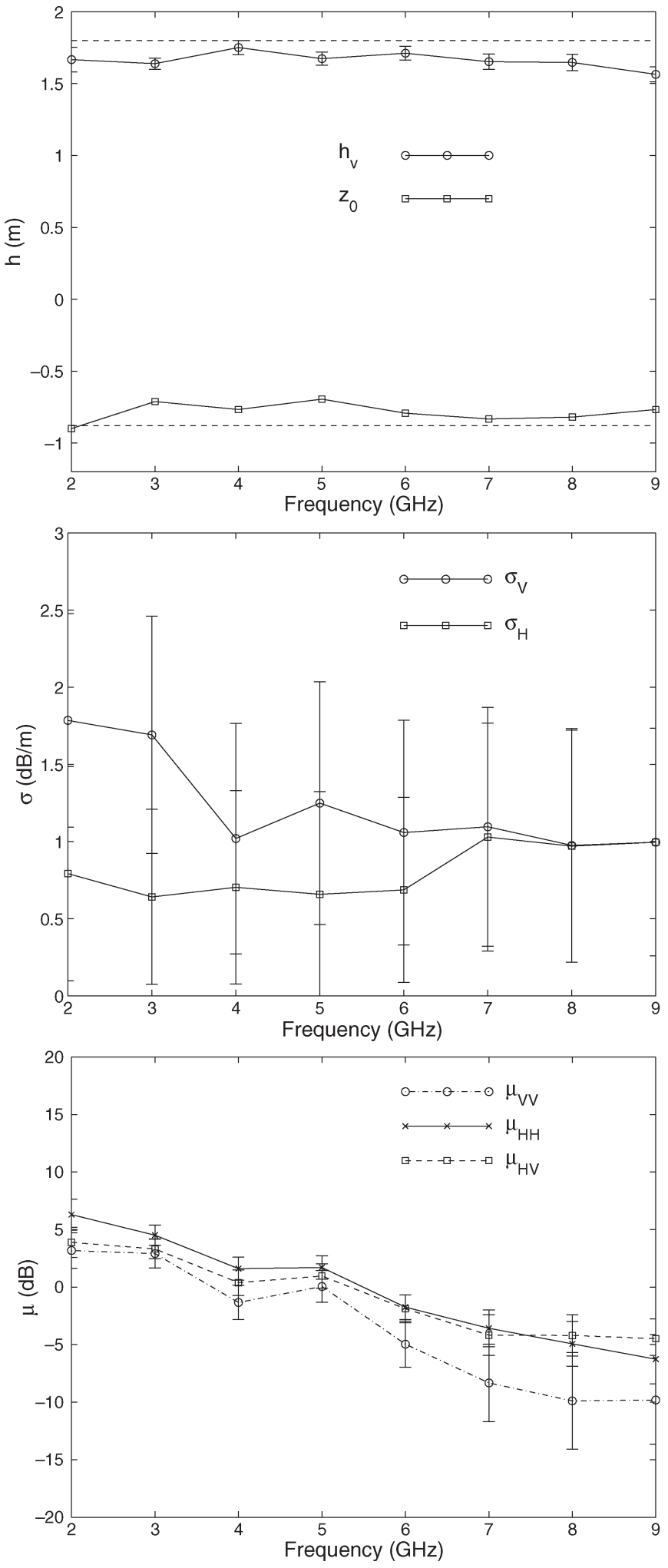

Fig. 6. Inversion results with the hybrid approach for the maize sample as a function of frequency. $B=0.25^{\circ}$, and $k_{z}=0.26 \cdot f$ (in gigahertz).

ing. The minimization of the global distance between model coherences and data has been implemented with a genetic algorithm named GENOCOP2 [17].

The results obtained for the maize case with $B=0.25^{\circ}$ $\left[k_{z}=0.26 \cdot f(\mathrm{GHz})\right]$ are shown in Fig. 6 as a function of 
frequency from 2 to $9 \mathrm{GHz}$. Due to the high nonlinearity of the equations' system, all solutions suffer from a high dependence upon the initial guess. We have carried out 100 realizations of the inversion solution for each frequency, providing the algorithms with random initial values. Consequently, results are plotted in the form of mean values and standard deviations, thus providing quantitative measurements about the stability or robustness of the inversion.

The estimation of vegetation height and topography is uniform with the frequency and presents a low variability. On the other hand, extinction estimates are quite variable (see their standard deviation), although their mean values agree with the predictions of the electromagnetic theory: Vertical extinction is higher than horizontal one, and they approach each other as frequency increases. The interpretation of the retrieved values of ground-to-volume ratios is not evident, since many parameters contribute to their final values [6]. As a general comment, their absolute values decrease with increasing frequencies due to the stronger response from the aboveground vegetation volume at higher frequencies. Moreover, as expected, $\mu_{\mathrm{VV}}$ remains always below the other two channels, because of the weaker response from the ground-stem double-bounce contribution.

It is interesting to mention the importance of the first step in this approach: estimation of the topographic phase. By reviewing the comparison between Fig. 3(c) and (d), we concluded that, for enclosing all coherences, it was necessary to reduce the vegetation height $h_{\mathrm{v}}$ and to increase the maximum extinction $\sigma_{\max }$. In addition, with these new values of $h_{\mathrm{v}}$ and $\sigma_{\max }$, the location of coherences, when projected onto the lines in Fig. 3(d), is farther from the unit circumference point $\exp \left(j \phi_{0}\right)$ than in Fig. 3(c). In other words, all coherences correspond to lower values of ground-to-volume ratio $\mu$ than before. Analogously, an error in $\phi_{0}$ would produce a shift in the parameters required to enclose the coherences with the extreme lines $\left(h_{\mathrm{v}}\right.$ and $\left.\sigma_{\max }\right)$ and in their corresponding $\mu$. For example, with the same interferometer configuration, if one estimates a ground position lower than the actual topography, the position of the estimated $\phi_{0}$ will move clockwise with respect to the true one. As a result, if one draws the necessary extreme lines for $\sigma=0$ and $\sigma_{\max }$ and with a shorter $h_{\mathrm{v}}$, the coherences will correspond to lower values of $\mu$ than in the case of a right estimation of $\phi_{0}$. Consequently, the position of $\phi_{0}$ very significantly affects the rest of the parameters. If we translate this argumentation to the hybrid inversion algorithm, a wrong estimation of $\phi_{0}$ would significantly change the solution of the remaining six unknowns. Nevertheless, the ground topography is calculated by using the whole set of data, and its inversion works very well in all experiments treated in this paper, as observed by the accuracy shown for $z_{0}$ in the results of the hybrid approach, with respect to the true ground position. As discussed in later sections, this accuracy is in contrast with the performance of the dual-baseline approach proposed later, particularly for the rice sample.

Fig. 7 shows the estimates obtained with the same target but with a doubled baseline $\left(B=0.5^{\circ}, k_{z}=0.52 \cdot f(\mathrm{GHz})\right.$ ). As discussed in [9], when using the larger baseline, the extreme volume decorrelation above $6 \mathrm{GHz}$ makes the estimates
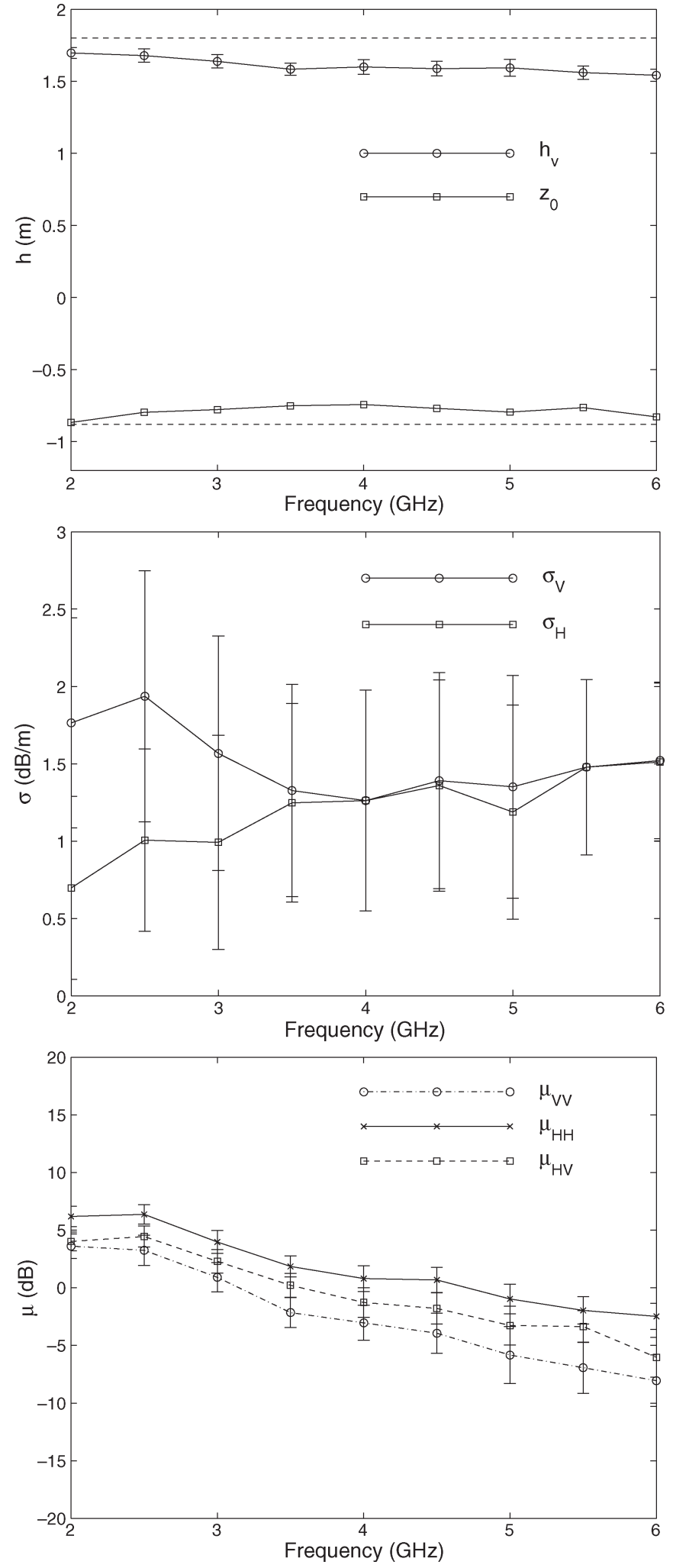

Fig. 7. Inversion results with the hybrid approach for the maize sample as a function of frequency. $B=0.5^{\circ}$, and $k_{z}=0.52 \cdot f$ (in gigahertz).

meaningless, so they are not included in this figure. It can be observed that the estimation of the vegetation height and topography is quite stable with the frequency for this baseline too, and values are quite similar for both baselines. However, 
vertical and horizontal extinctions approach each other faster than with the short baseline, but maintaining the same large variability. Finally, ground-to-volume ratios show the same trend and relative ordering as before.

The discrepancies of the retrieved results with the actual values are a consequence of the data-model mismatch shown in Fig. 3. Note that, in the particular cases in Figs. 3(d) and (e) and 4(a), certain volume height values for a correct fitting were chosen, and these values correspond to those contained in the standard-deviation interval shown in the retrieved estimates in Figs. 6 and 7 at $3 \mathrm{GHz}$ for a $0.5^{\circ}$ baseline and at $6 \mathrm{GHz}$ for a $0.25^{\circ}$ baseline, respectively.

We have applied the same inversion strategy to the rice sample. Figs. 8 and 9 show the obtained estimates for the frequency range 5-9 $\mathrm{GHz}$ with baselines of $0.5^{\circ}\left[k_{z}=0.52\right.$. $f(\mathrm{GHz})]$ and $1^{\circ}\left[k_{z}=1.04 \cdot f(\mathrm{GHz})\right]$, respectively. The radar response from this target is dominated by the backscattering from the ground-stem interaction, which is helped by the flooded condition of the soil. This dominance produces two important effects. First, below $5 \mathrm{GHz}$, the backscatter response of the short vegetation volume is too weak, when compared to the ground-stem contribution, to produce any useful features in the PolInSAR observables and, as a result, to invert any parameter from them. Second, all coherences are very concentrated on the complex plane, forming a cluster close to the unit circumference.

The proximity to the unit circumference yields extremely accurate estimates of the ground position, since any fitted line crosses the true topographic phase $\phi_{0}$. Note that the interferometric phase of the ground-stem response is located exactly at the air-ground interface. This line fitting provides errors below $1 \mathrm{~cm}$ in the whole frequency range for the topography. The counterpart of the aforementioned disposal of the coherences is the reduced size of the visible region, which complicates the numerical inversion of the remaining six parameters. The estimation of vegetation height behaves well above $6 \mathrm{GHz}$. The average of the estimated values is closer to the true height $(75 \mathrm{~cm})$ for the $1^{\circ}$ baseline, but their variance is larger than that for the short baseline. The interpretation of the retrieved extinctions is very difficult from the physical point of view. In our opinion, the estimates are so unstable due to two related causes: the low sensitivity of the direct model to extinction, already described in Section III, and the low influence of extinction on the signal because of the short vegetation volume (only $75 \mathrm{~cm}$ ). Finally, the trend of the ground-to-volume ratios agrees with the observations made before for the maize target but not their relative ordering. In the case of the rice, the flooded condition of the soil, which produces an extremely flat air-ground interface, significantly reduces the crosspolar contribution from the ground-stem interaction. As a result, the lowest ground-tovolume ratio is the crosspolar one. At the other extreme, as for the maize case, $\mu_{\mathrm{HH}}$ is the highest. However, $\mu_{\mathrm{VV}}$ is very close to $\mu_{\mathrm{HH}}$ at the highest frequencies, when the vegetation volume backscattering becomes significant.

Also note that, similar to the maize results, the height chosen for the data-model fitting in Fig. 4(b) is contained in the standard-deviation interval in the retrieved results shown in Fig. 8 at $8 \mathrm{GHz}$.
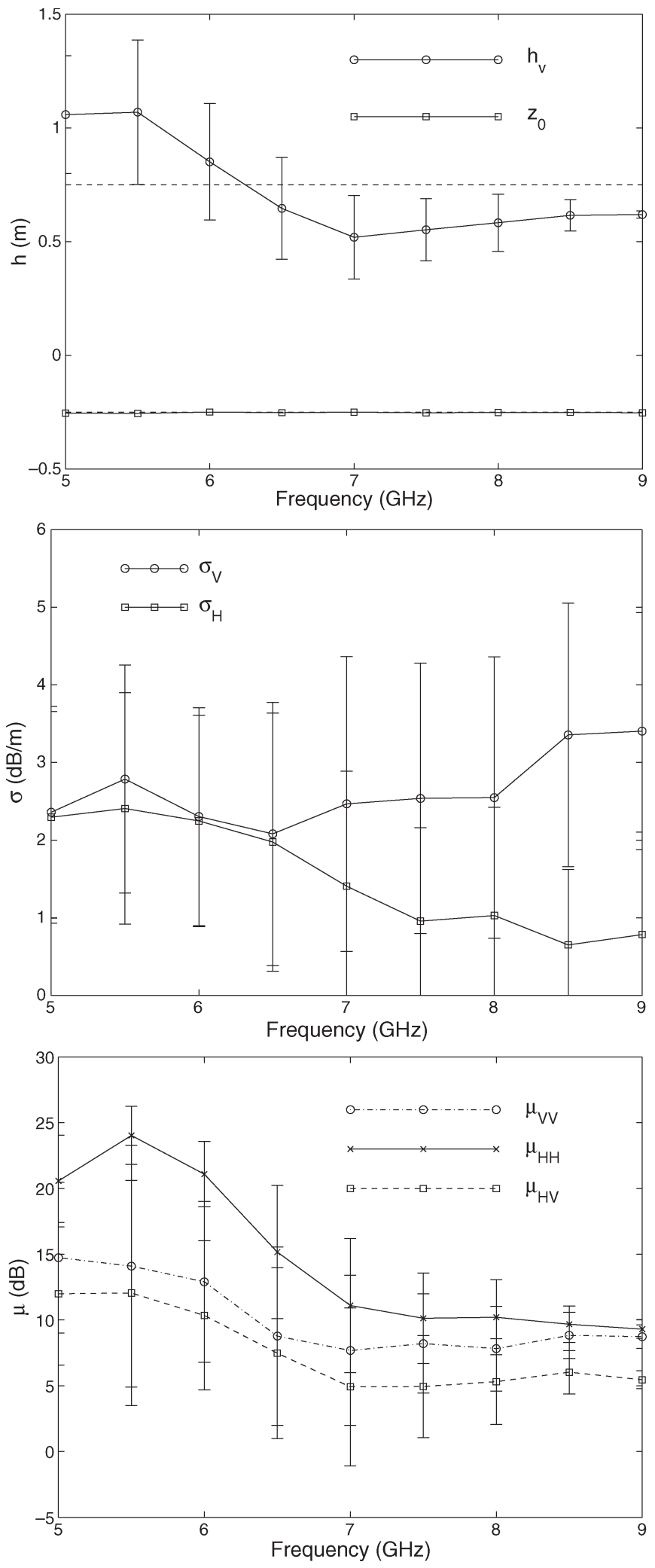

Fig. 8. Inversion results with the hybrid approach for the rice sample as a function of frequency. $B=0.5^{\circ}$, and $k_{z}=0.52 \cdot f$ (in gigahertz).

\section{B. Dual-Baseline Approach}

The second inversion technique proposed in this paper is based on increasing the observation space by adding a second interferometric baseline. With two baselines, we have 12 input 

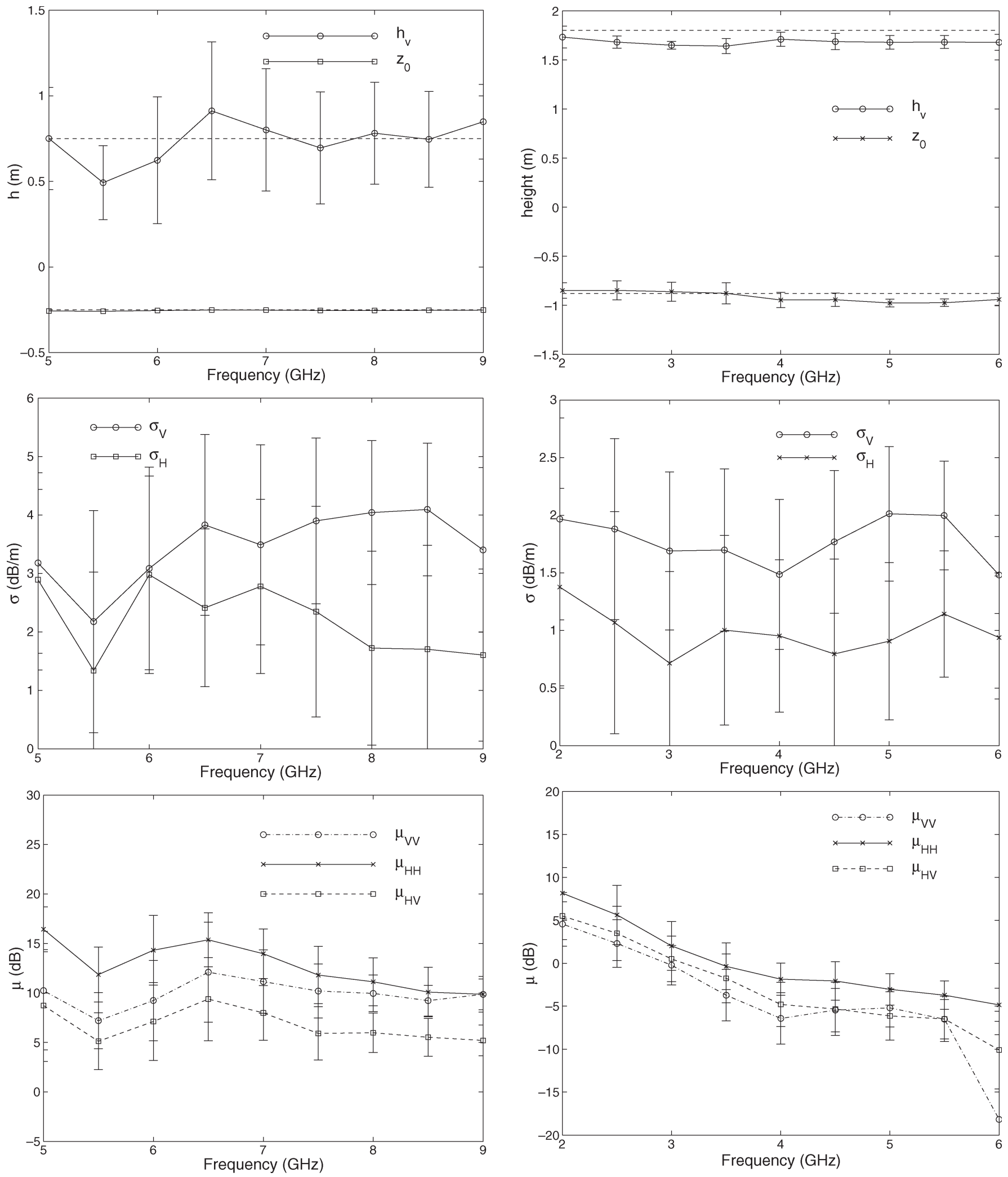

Fig. 9. Inversion results with the hybrid approach for the rice sample as a function of frequency. $B=1^{\circ}$, and $k_{z}=1.04 \cdot f$ (in gigahertz).

Fig. 10. Inversion results with the dual-baseline approach for the maize sample as a function of frequency. $B=0.25^{\circ}-0.5^{\circ}$.

real data available to estimate seven model unknowns. The solution of the model fitting has been implemented with a genetic algorithm as well.
We show in Fig. 10 the results obtained for the maize sample from 2 to $6 \mathrm{GHz}$. The maximum frequency has been limited to avoid the volume decorrelation produced above $6 \mathrm{GHz}$ by the 
$0.5^{\circ}$ baseline. The ground topography and the vegetation height are estimated quite precisely in the whole frequency range. These height estimates are also very stable from the numerical point of view. In contrast, extinction estimates fluctuate when we change the initial solution of the algorithm (see the error bars). Nevertheless, as in the results from the hybrid algorithm, vertical extinction is always higher than the horizontal one. Their difference ranges between 0.5 and $1 \mathrm{~dB} / \mathrm{m}$. Finally, all ground-to-volume ratios significantly decrease with frequency, as we expected from the stronger vegetation response at higher frequencies.

Fig. 11 shows the estimates of the dual-baseline approach applied to the rice data from 5 to $9 \mathrm{GHz}$. Frequencies below $5 \mathrm{GHz}$ are discarded because the direct backscattering from the stems and leaves is too weak when compared to the ground-stem interaction [9]. The ground position $z_{0}$ is retrieved with extremely good accuracy (maximum absolute error below $1 \mathrm{~cm}$ ), as a result of the dominance of the ground-stem contribution, which is emphasized by the flooded condition of the soil. Plants' height is slightly underestimated, and it presents a higher variance than in the maize case. Note that, in this case, due to the short vegetation volume, the visible region of the coherences is reduced to a small cluster, and hence, a greater ambiguity in the height estimation is produced. Extinction estimates are less stable than in the maize experiment, and the average values of $\sigma_{\mathrm{V}}$ and $\sigma_{\mathrm{H}}$ do not approach each other at high frequencies. All ground-to-volume ratios are quite constant with frequency, and $\mu_{\mathrm{HV}}$ is always the smallest due to its lowest ground-stem response because of the ground flatness, as previously explained.

Retrieved values for the ground-to-volume ratios are different for the rice when obtained by the hybrid approach (see Figs. 8 and 9) and by the dual-baseline algorithm (see Fig. 11). More precisely, all $\mu$ estimates for the dual baseline are lower than for the hybrid approach (note that the vertical scales are different). Their difference ranges from 20 to $5 \mathrm{~dB}$, depending on the frequency and the polarization channel. In addition, the estimated vegetation heights in Fig. 11 are mostly lower than with the hybrid approach. The slightly different performance of both techniques is a consequence of their working principle. As explained in the previous section, the first step in the hybrid approach provides a good approximation of the ground topography $z_{0}$, whose accuracy influences the rest of the unknowns. With the dual-baseline algorithm, all seven parameters are estimated at the same time, and none of them exhibits any preference. Consequently, the numerical minimization algorithm is more likely to fall in local minima with ground positions lower than the true one, and as explained, in these cases, the rest of the inverted parameters suffer the corresponding change: All $\mu$ 's move to lower values, and $h_{\mathrm{v}}$ is also smaller. We have observed this specific behavior in the detailed results, showing the presence of a number of solutions with these properties: lower values of $z_{0}, h_{\mathrm{v}}$, and $\mu$. Note that the error in these estimates of $z_{0}$ is about $2-5 \mathrm{~cm}$ below the true ground position. This small offset is enough to significantly alter the rest of the parameters in the case of the rice because all coherences are very close to the unit circumference.
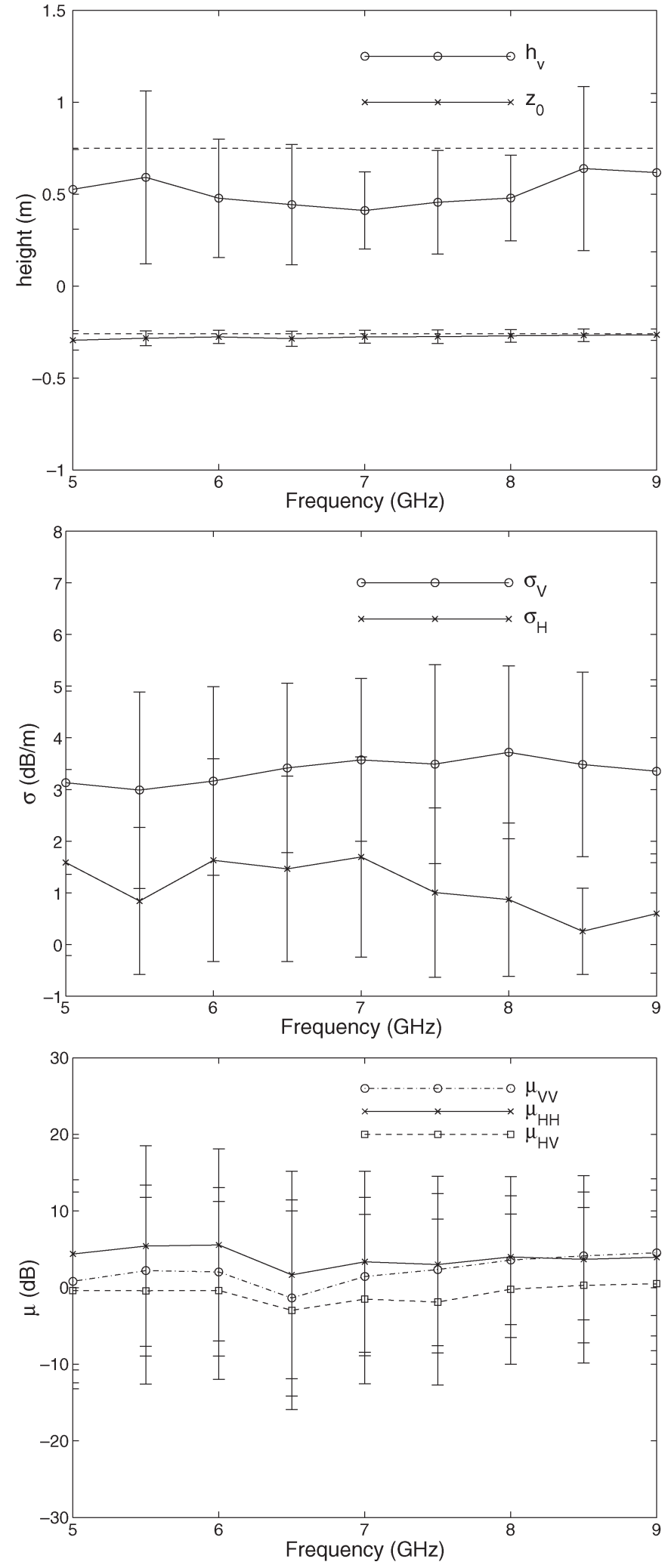

Fig. 11. Inversion results with the dual-baseline approach for the rice sample as a function of frequency. $B=0.5^{\circ}-1^{\circ}$.

The presence of the mentioned shifted solutions within the whole set of solutions (obtained with different initializations) produces a decrease in the average estimation of ground-tovolume ratios and vegetation depth. A second consequence is an 


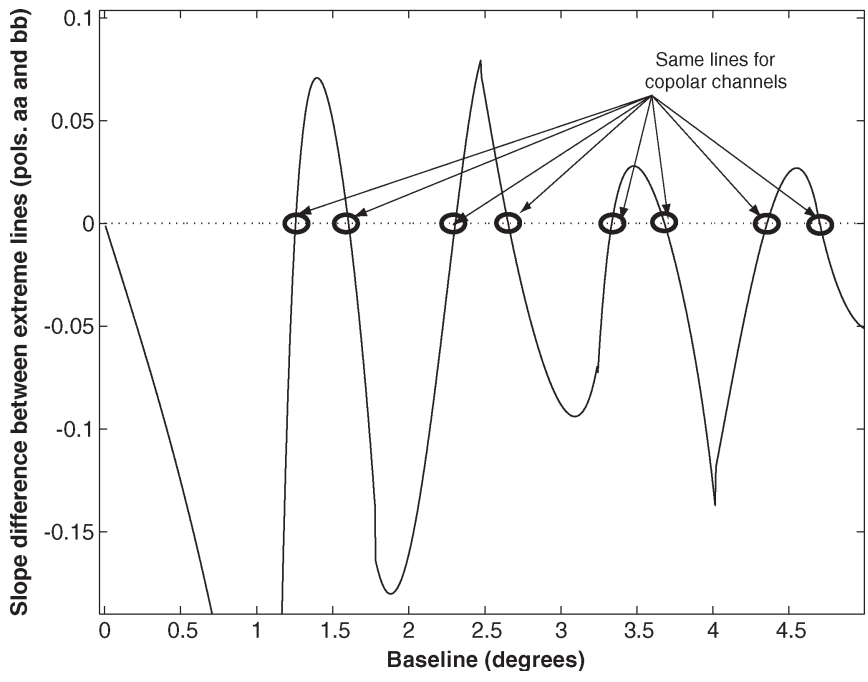

Fig. 12. Baseline effect on the width of the coherence region. Plot of the slope difference between extreme lines (copolar channels) of the coherence region. Parameters: $k_{z}=3.1 \cdot B$ (in degrees), $f=3 \mathrm{GHz}, \sigma_{a}=3 \mathrm{~dB} / \mathrm{m}$, $\sigma_{b}=0.5 \mathrm{~dB} / \mathrm{m}, \theta_{0}=45^{\circ}, z_{0}=-70 \mathrm{~cm}$, and $h_{\mathrm{v}}=2 \mathrm{~m}$. (Circles) Zero slope difference values correspond to baselines that make the coherence region become a single line.

increase in the variability of the results, which is quite evident in the standard deviations of the ground-to-volume ratios.

Despite these drawbacks, note that the dual-baseline approach enables the estimation of the whole set of parameters simultaneously by means of a numerical optimization algorithm. In order to take advantage of such feature, i.e., there is no need to have coherence points well separated along the line, the adaptation of the current OVoG model to take into account a more realistic backscattering response, i.e., not exponential [12], is mandatory.

Additionally, it must be pointed out that the dual-baseline approach deserves a deeper analysis in order to describe more precisely the impact of the baseline ratio in the inversion performance. As first step, a number of simulations have been carried out as a function of the baseline in order to explore the effect on the coherence loci. First observations show that the separation between the extreme lines (for the copolar channels) that define the region of coherences is not a monotonic function of the baseline and reaches a zero angle for certain baselines. This feature is illustrated in Fig. 12, where difference of slopes between copolar lines is plotted as a function of the baseline. Based on this configuration, positive slope differences correspond to an inversion of the relative positions of extreme lines of the coherence region with respect to the ones predicted by the model physics (i.e., the line of the copolar channel with the highest extinction should be located closer to the origin). Zero-slope difference values correspond to a collapse into a single line.

This variation of the width of the coherence region as a function of the baseline, together with the increasing volume decorrelation for larger baselines, must be taken into account for inversion purposes. On one hand, the coherence region at a certain baseline should be wide enough to assure some sensitivity for extinction. On the other hand, a narrow region is useful for an accurate topography estimation, which is a key parameter significantly affecting the retrieval of the rest of the parameters.

\section{CONCLUSION}

Experimental PolInSAR data gathered in laboratory conditions and direct model predictions have been compared in this paper. This comparison has been useful in testing the fidelity of the RVoG and OVoG models with respect to two agricultural samples. An overall agreement between the model and the data has been found, but a partial mismatch in the ordering of the coherence positions on the complex plane as a function of extinction has been detected. In addition, the shape and location of the visible region on the complex plane does not fit exactly with the feasible region predicted by the model, unless a tuning in the scene parameters is carried out. Finally, this comparison has pointed out the low sensitivity of the direct model to the extinction coefficients.

The discrepancies between the model and experimental data have been justified by two simplifications assumed in the model formulation, more precisely, the homogeneity in the vertical structure of the vegetation volume and the exclusion of multiple scattering terms in the electromagnetic background. However, owing to the simplicity of the model, its inversion is possible.

The second part of this paper has been devoted to present two strategies for the inversion of the full set of parameters of the OVoG model: a geometrical-numerical approach and a dual-baseline approach. Both techniques have shown good results in the estimation of the ground topography and the vegetation height, but a very varying estimation of the extinction coefficients. The dual-baseline approach, which delivers a simultaneous estimation of the whole set of parameters, has shown slightly worse estimates in the case of the rice, since it significantly suffers more from the influence of the initial solution values in the numerical minimization. On the contrary, the hybrid approach is more robust due to a better estimation of the ground topography, which is independently obtained from the rest of the parameters. Further research, based on numerical simulations, must be carried out in order to show the impact of the baseline values, and the baseline ratio in the dual-baseline case, in the inversion performance.

Both analyses (direct model and inversion approaches) are founded on experimental data acquired in an anechoic chamber, so a more realistic comparison is expected to be performed with the other data to be obtained with air- or space-borne systems. In this context, the requirement of a single-pass configuration should be highlighted, in order to cancel the strong temporal decorrelation expected in this kind of vegetation due to the fast crop development.

\section{ACKNOWLEDGMENT}

The authors would like to thank the personnel of the EMSL for their support in performing the measurements and providing the experimental data. The genetic algorithm GENOCOP2 [17] was developed by Dr. Z. Michalewicz and is freely available at www.coe.uncc.edu/ zbyszek/evol-systems.html. 


\section{REFERENCES}

[1] P. Dubois-Fernandez, S. Angelliaume, J.-C. Souyris, F. Garestier, and I. Champion, "The specificity of P band PolInSAR data over vegetation," in Proc. 3rd PolInSAR Workshop, Frascati, Italy, Jan. 2007. [Online]. Available: http://earth.esa.int/workshops/polinsar2007.

[2] T. Mette, K. P. Papathanassiou, and I. Hajnsek, "Applying a common allometric equation to convert forest height from Pol-InSAR data to forest biomass," in Proc. IGARSS, Anchorage, AK, Sep. 2004, vol. 1, pp. 269-272.

[3] T. Mette, K. P. Papathanassiou, and I. Hajnsek, "Biomass estimation from polarimetric SAR interferometry over heterogeneous forest terrain," in Proc. IGARSS, Anchorage, AK, Sep. 2004, vol. 1, pp. 511-514.

[4] F. Garestier, P. Dubois-Fernandez, X. Dupuis, P. Paillou, and I. Hajnsek, "PolInSAR analysis of X-Band data over vegetated and urban areas," IEEE Trans. Geosci. Remote Sens., vol. 44, no. 2, pp. 356-364, Feb. 2006.

[5] R. N. Treuhaft, S. N. Madsen, M. Moghaddam, and J. J. van Zyl, "Vegetation characteristics and underlying topography from interferometric radar," Radio Sci., vol. 31, no. 6, pp. 1449-1485, Nov. 1996.

[6] R. N. Treuhaft and P. R. Siqueira, "Vertical structure of vegetated land surfaces from interferometric and polarimetric data," Radio Sci., vol. 35, no. 1, pp. 141-177, 2000.

[7] S. R. Cloude and K. P. Papathanassiou, "Three-stage inversion process for polarimetric SAR interferometry," Proc. Inst. Electr. Eng.-Radar, Sonar Navig., vol. 150, no. 3, pp. 125-134, Jun. 2003.

[8] R. N. Treuhaft and S. R. Cloude, "The structure of oriented vegetation from polarimetric interferometry," IEEE Trans. Geosci. Remote Sens., vol. 37, no. 5, pp. 2620-2624, Sep. 1999.

[9] J. D. Ballester-Berman, J. M. Lopez-Sanchez, and J. Fortuny-Guasch, "Retrieval of biophysical parameters of agricultural crops using polarimetric SAR interferometry," IEEE Trans. Geosci. Remote Sens., vol. 43, no. 4, pp. 683-694, Apr. 2005.

[10] I. Hajnsek and S. R. Cloude, "Pol-InSAR for agricultural vegetation parameter estimation," in Proc. IGARSS, Anchorage, AK, Sep. 2004, vol. 2, pp. 1224-1227.

[11] S. R. Cloude and K. P. Papathanassiou, "Polarimetric SAR interferometry," IEEE Trans. Geosci. Remote Sens., vol. 36, no. 5, pp. 1551-1565, Sep. 1998.

[12] S. R. Cloude, "Dual baseline coherence tomography," IEEE Geosci. Remote Sens. Lett., vol. 4, no. 1, pp. 127-131, Jan. 2007.

[13] K. P. Papathanassiou and S. R. Cloude, "Single baseline polarimetric SAR interferometry," IEEE Trans. Geosci. Remote Sens., vol. 39, no. 11, pp. 2352-2363, Nov. 2001.

[14] F. T. Ulaby, A. Tavakoli, and T. B. A. Senior, "Microwave propagation constant for a vegetation canopy with vertical stalks," IEEE Trans. Geosci. Remote Sens., vol. GRS-25, no. 6, pp. 714-725, Nov. 1987.

[15] L. Tsang, K. Ding, G. Zhang, and J. A. Kong, "Backscattering enhancement and clustering effects of randomly distributed dielectric cylinders overlying a dielectric half space based on Monte Carlo simulations," IEEE Trans. Antennas Propag., vol. 43, no. 5, pp. 488-499, May 1995.

[16] S. M. C. Brown, S. Quegan, K. Morrison, J. C. Bennett, and G. Cookmartin, "High-resolution measurements of scattering in wheat canopies-Implications for crop parameter retrieval," IEEE Trans. Geosci. Remote Sens., vol. 41, no. 7, pp. 1602-1610, Jul. 2003.

[17] Z. Michalewicz, Genetic Algorithms + Data Structures = Evolution Programs, 3rd ed. New York: Springer-Verlag, 1996.

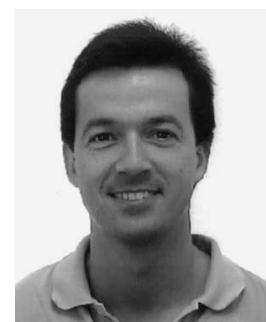

Juan M. Lopez-Sanchez (S'94-M'00-SM'05) was born in Alicante, Spain, in 1972. He received the Ingeniero and Doctor Ingeniero degrees in telecommunications engineering from the Technical University of Valencia, Valencia, Spain, in 1996 and 2000, respectively.

From 1998 to 1999 , he was as a Predoctoral Grantholder with the Space Applications Institute, Joint Research Centre of the European Commission, Ispra, Italy. Since 2000, he has been leading the Signals, Systems, and Telecommunication Group of the University of Alicante, Alicante, Spain, where he is an Associate Professor. His main research interests include analytical and numerical models for multiplescattering problems, microwave remote sensing for inversion of biophysical parameters, polarimetric techniques, and SAR imaging algorithms.

Dr. Lopez-Sanchez received the Indra award for the best Ph.D. thesis about radar in Spain in 2001.

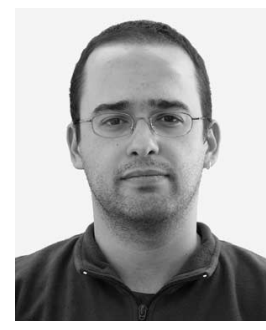

J. David Ballester-Berman was born in Xixona, Spain, in 1975. He received the Ingeniero degree in telecommunications engineering from the Technical University of Valencia, Valencia, Spain, in 2000 where he is currently working toward the Ph.D. degree.

From 2000 to 2001, he was a Support Engineer in the development of software tools for the planning and design of terrestrial digital television systems. Since 2001, he has been with the Signals, Systems, and Telecommunication Group of the University of Alicante, Alicante, Spain, as an Assistant Professor. His research interests include microwave remote sensing applied to biophysical parameter retrieval, electromagnetic modeling of vegetation covers, and radar imaging signal processing.

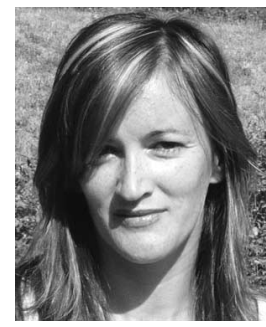

Yolanda Marquez-Moreno was born in Ciudad Real, Spain, in 1972. She received the Ingeniero degree in telecommunications engineering from the Technical University of Valencia, Valencia, Spain, in 1996, where she is currently working toward the Ph.D. degree.

Since 2000, she is with the Signals, Systems, and Telecommunication Group of the University of Alicante, Alicante, Spain, as a part-time Assistant Professor. Since 1997, she has been a Network Engineer with a telecommunication and broadcast company. Her research interests include applications of differential SAR interferometry and polarimetry. 